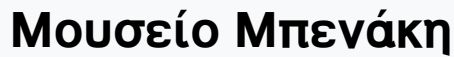

Tóp. 3 (2003)

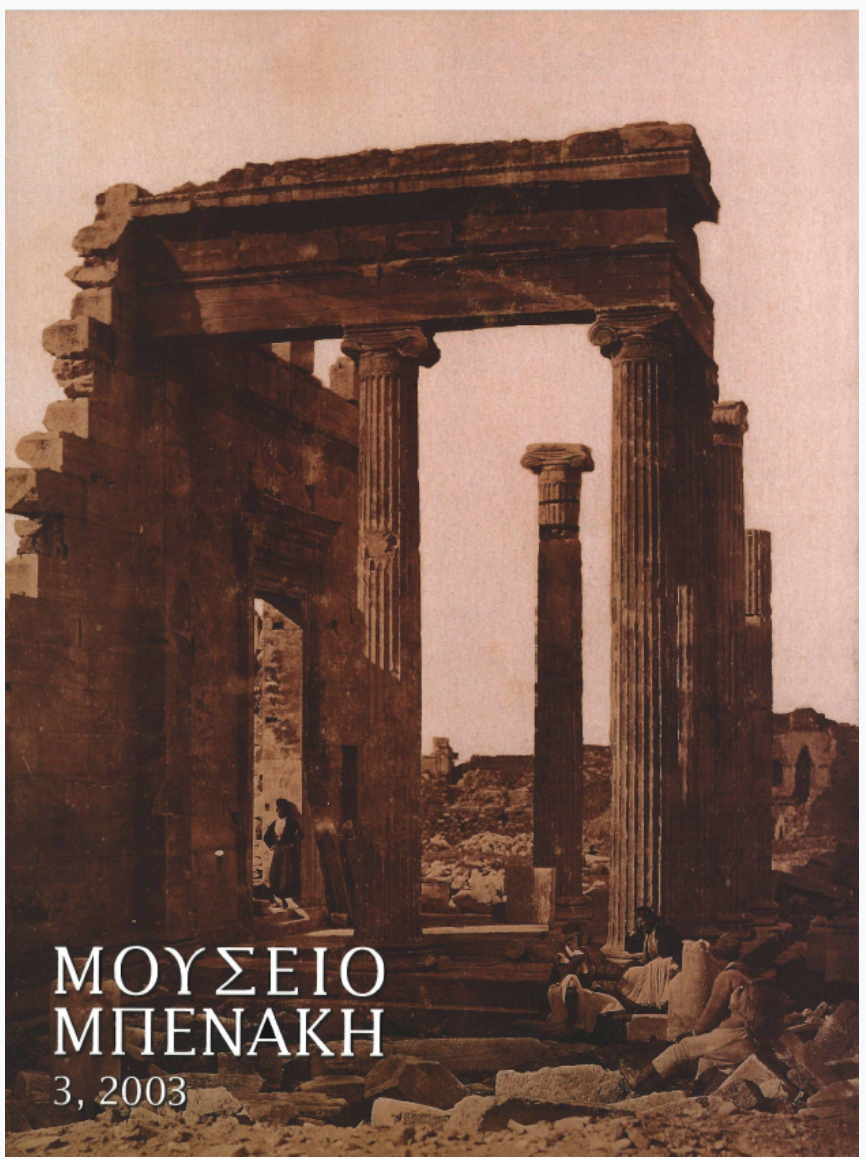

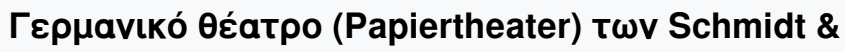
Romer anó tn $\Lambda \varepsilon \iota \psi i ́ a$

\section{Mapía Apyupıádn}

doi: $10.12681 /$ benaki.18242

Copyright @ 2018, Mapía Apyupıáōn

\section{(1) (1)(2)}

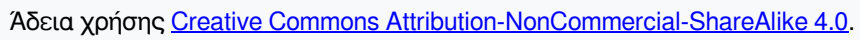

\section{Bıß入ıорачıкń avaчорá:}

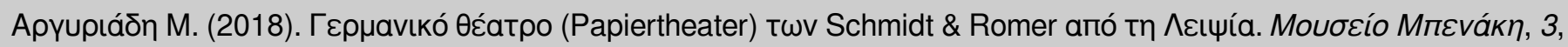
149-155. https://doi.org/10.12681/benaki.18242 


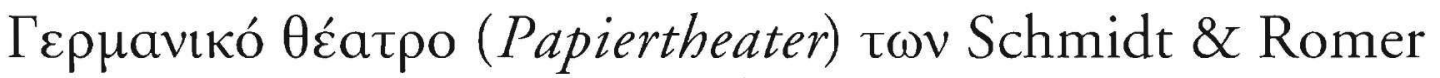

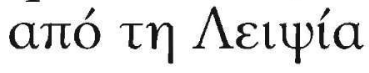

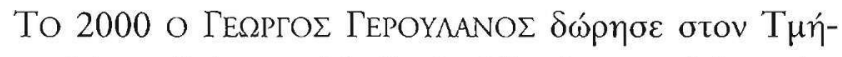
$\mu a$ Паıхvıเ

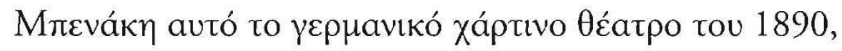

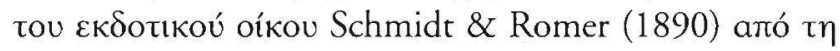

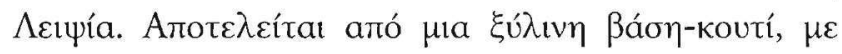

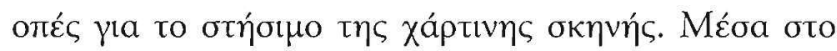

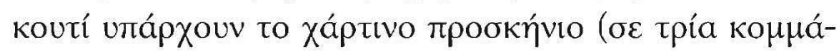

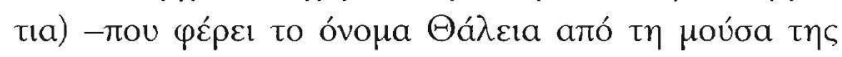

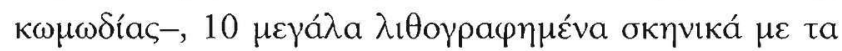

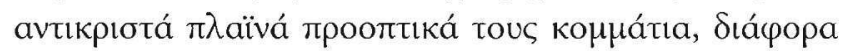

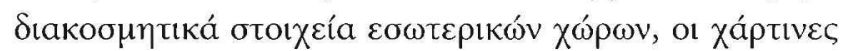

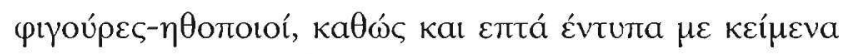

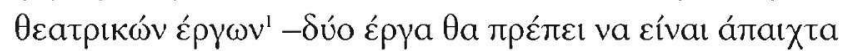

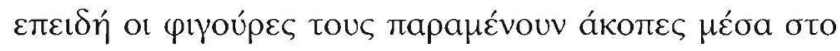
ह́vтuTó tous.

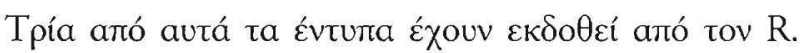

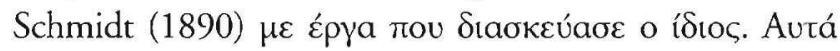

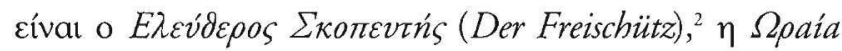

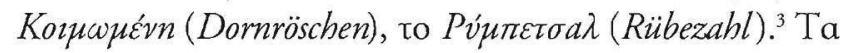

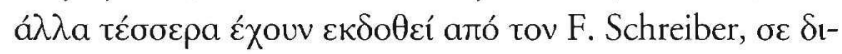

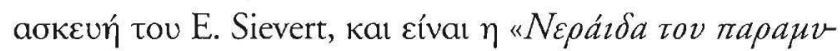

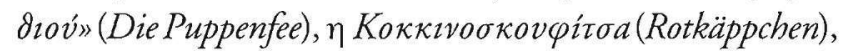

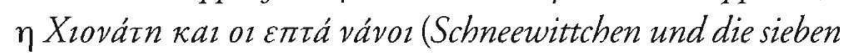

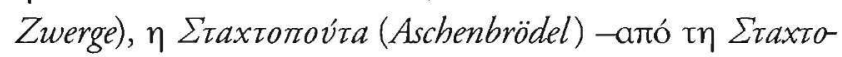

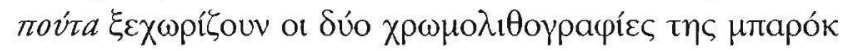

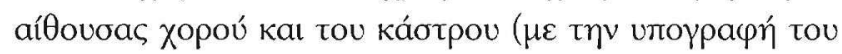

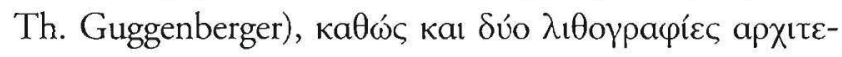

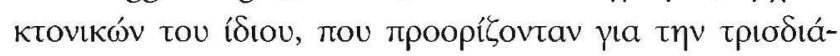

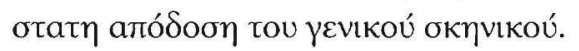

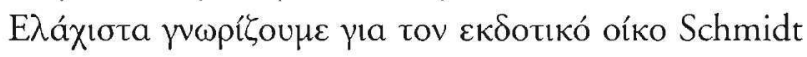

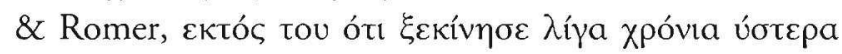

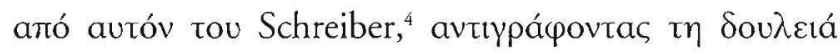

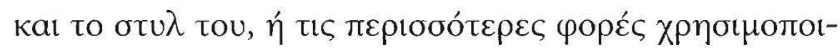

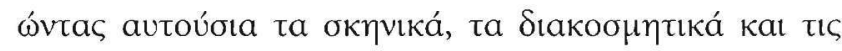

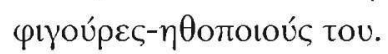

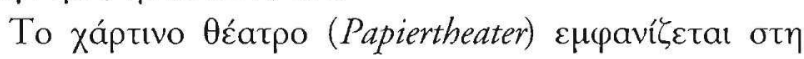

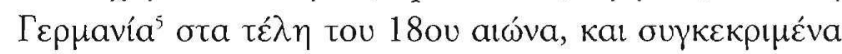

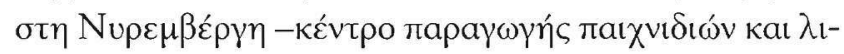

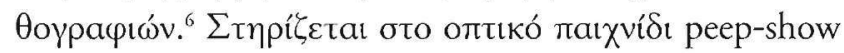

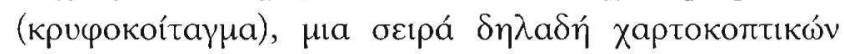

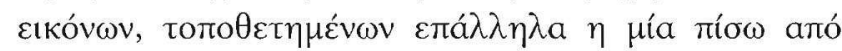

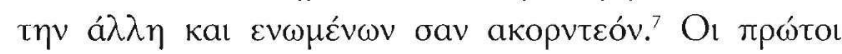

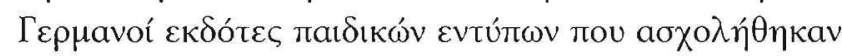

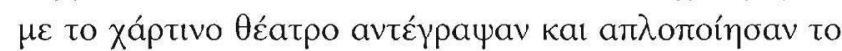

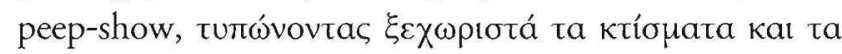

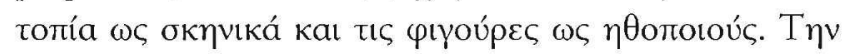

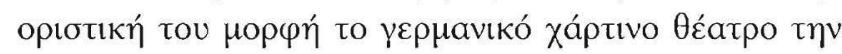

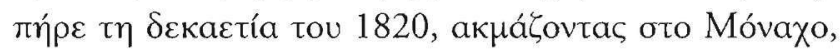

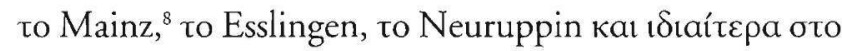

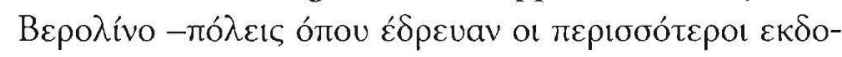

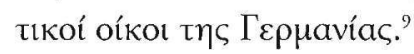

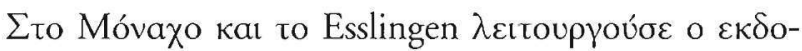

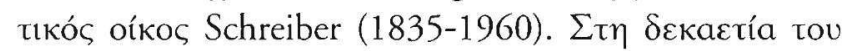
1850 o Ferdinand Schreiber $\mu \pi \eta ́ \kappa \varepsilon$ otov $\varepsilon \kappa \delta$ เıкó oúko

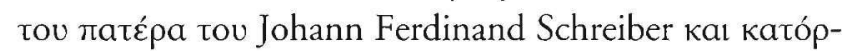

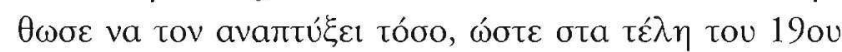
aı

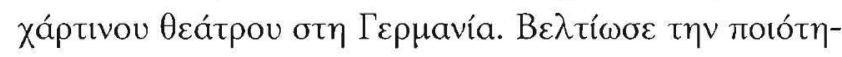

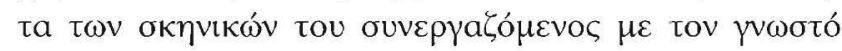

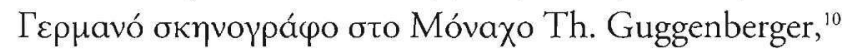

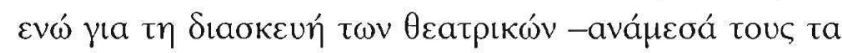

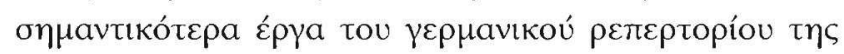




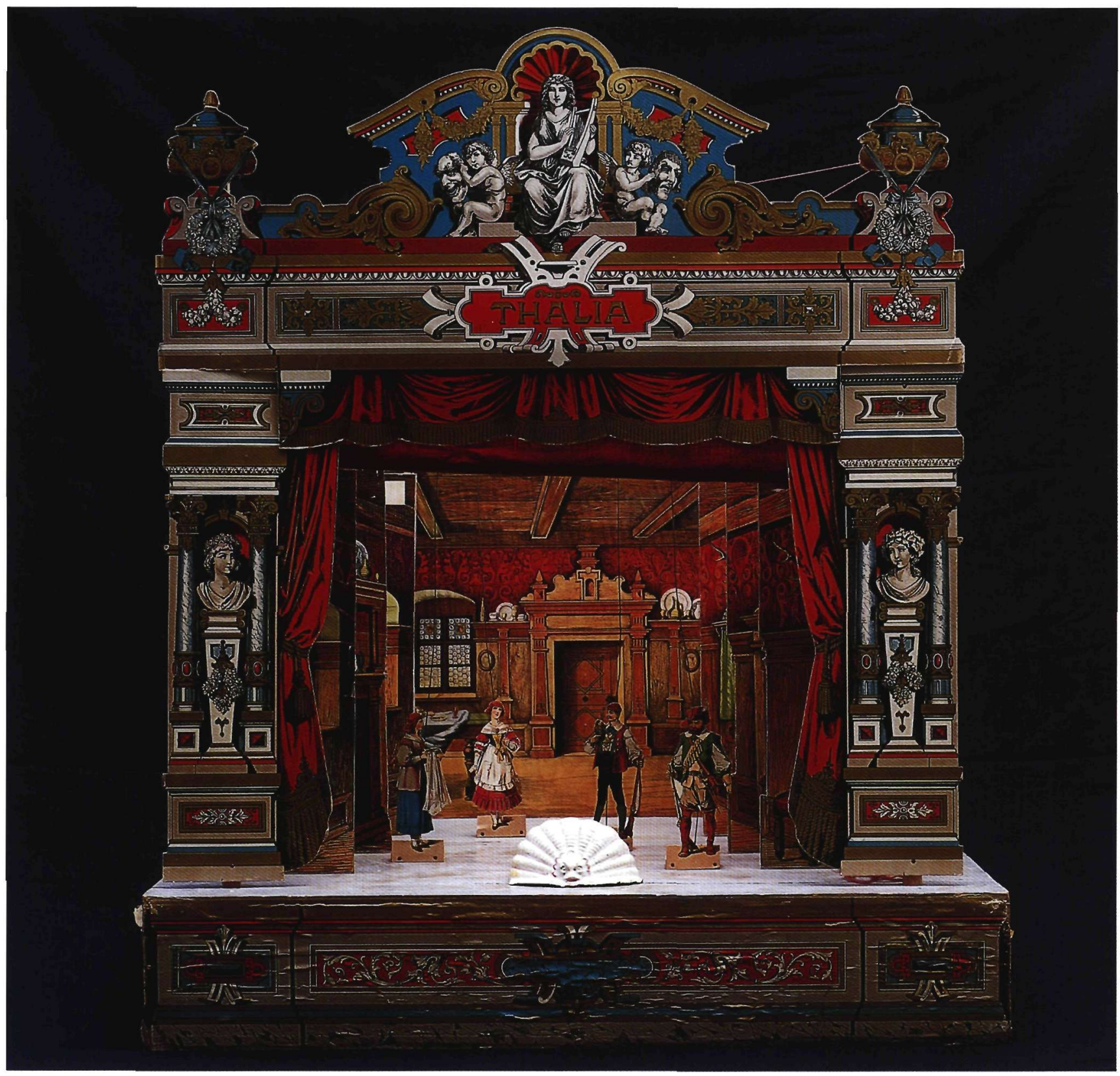

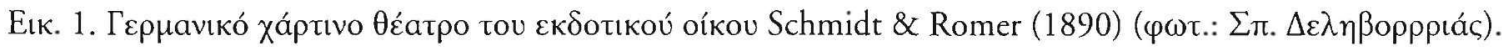

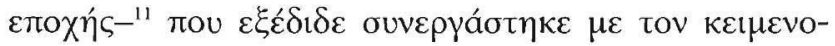

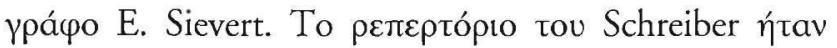

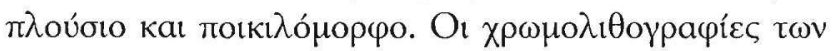

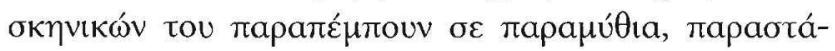

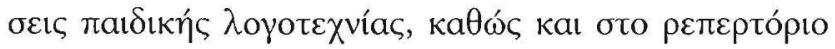

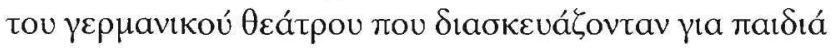

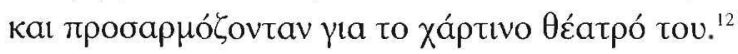

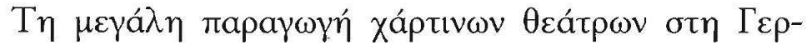

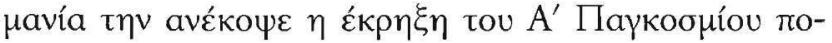

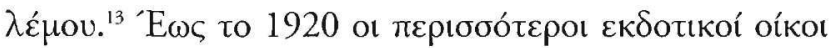

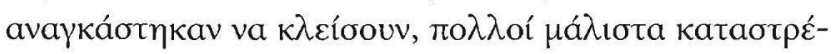

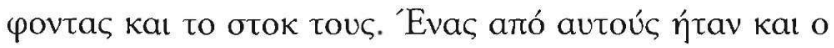

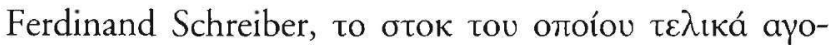

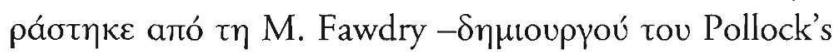

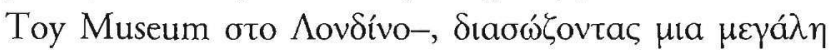

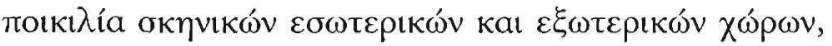




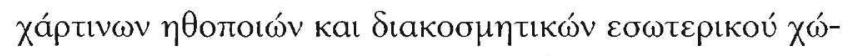

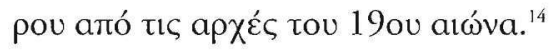

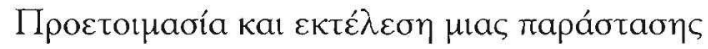

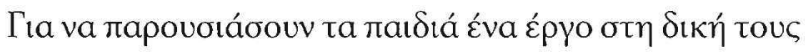

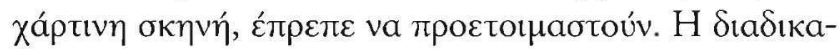

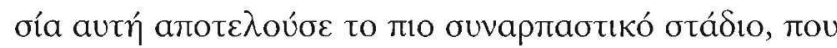

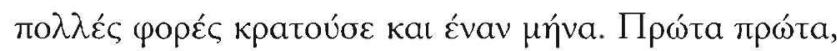

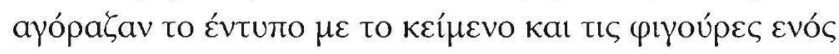

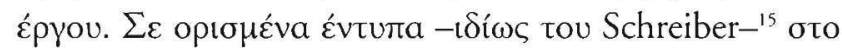

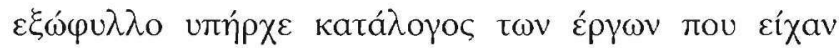

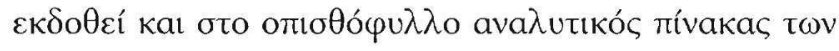

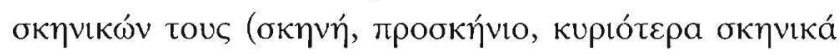

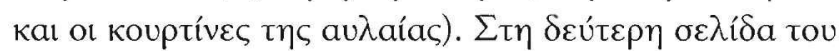

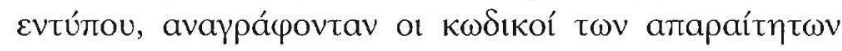

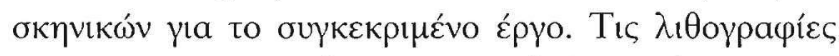

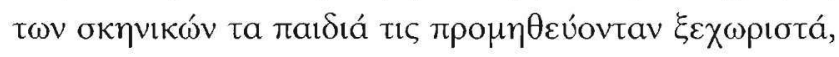

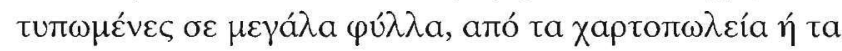

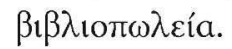

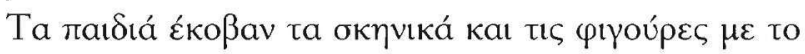

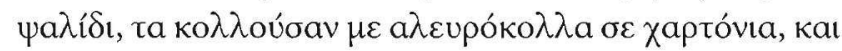

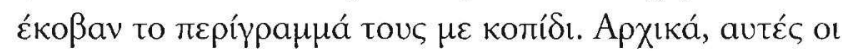

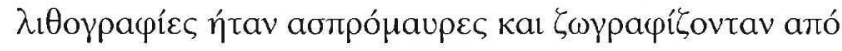

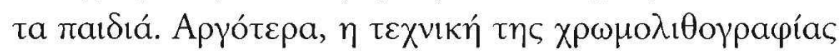

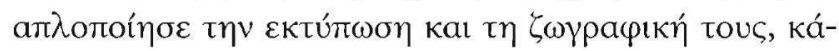

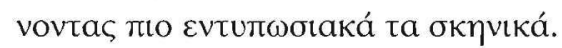

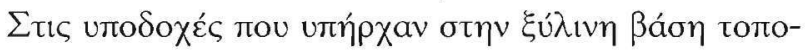

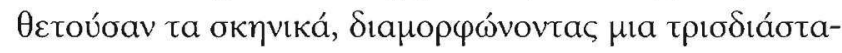

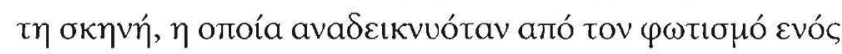

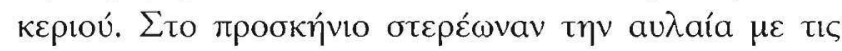

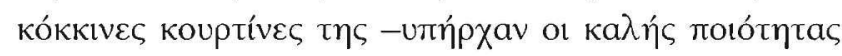

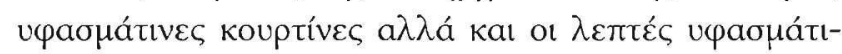

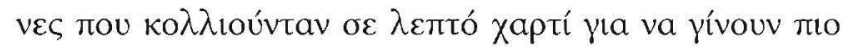

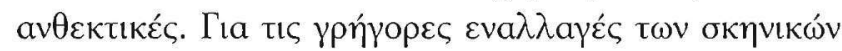

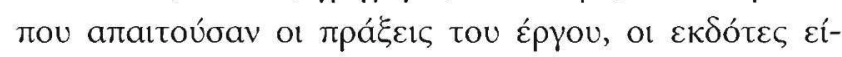

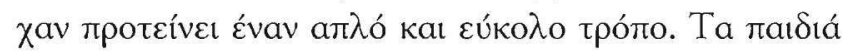

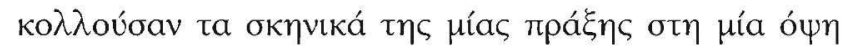

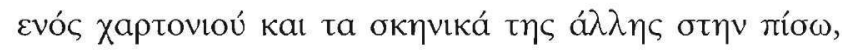

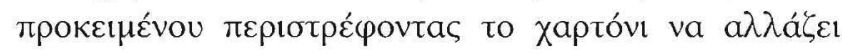

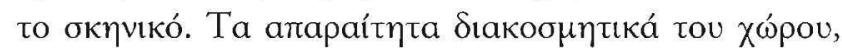

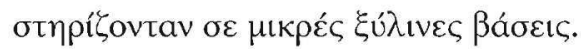

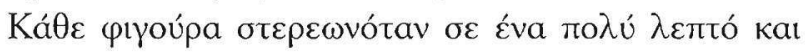

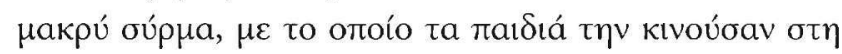

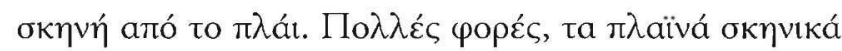

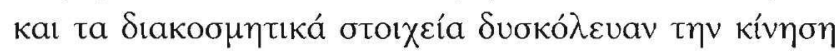

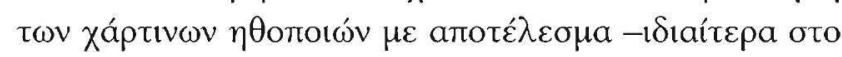
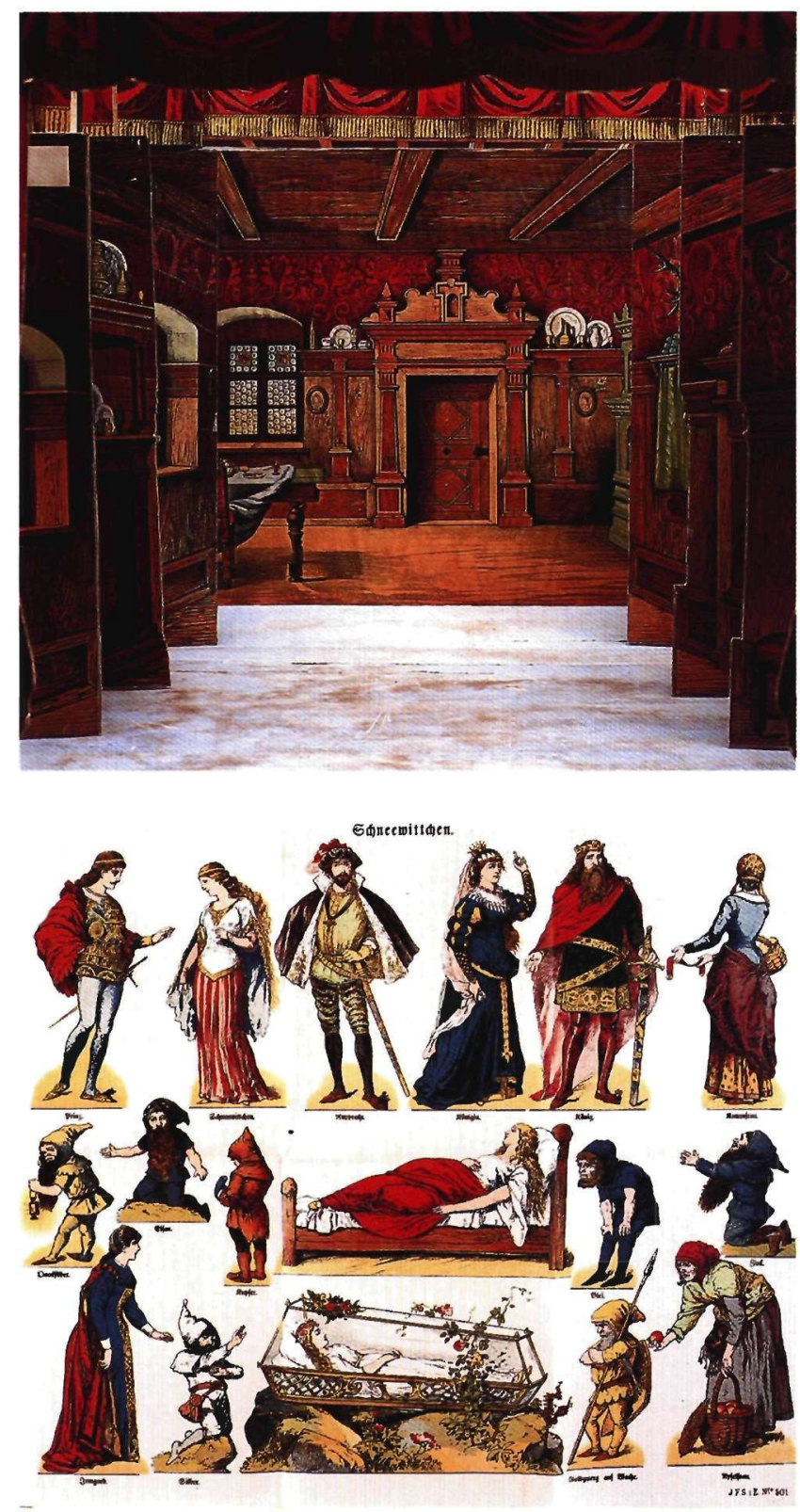

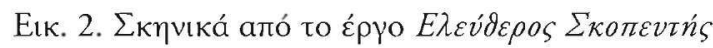

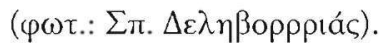

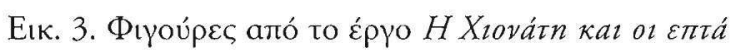
vávoน ( $\varphi \omega \tau .:$ $\Sigma \pi . \Delta \varepsilon \lambda \eta \beta о \rho \rho p เ a ́ c)$.

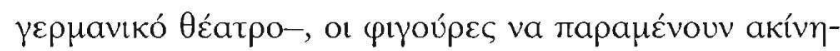

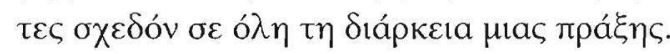

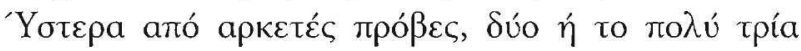

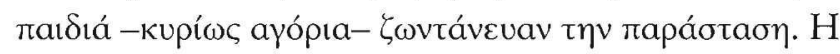

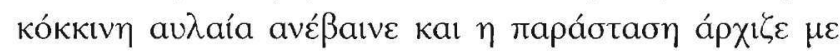

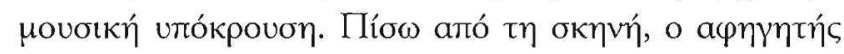




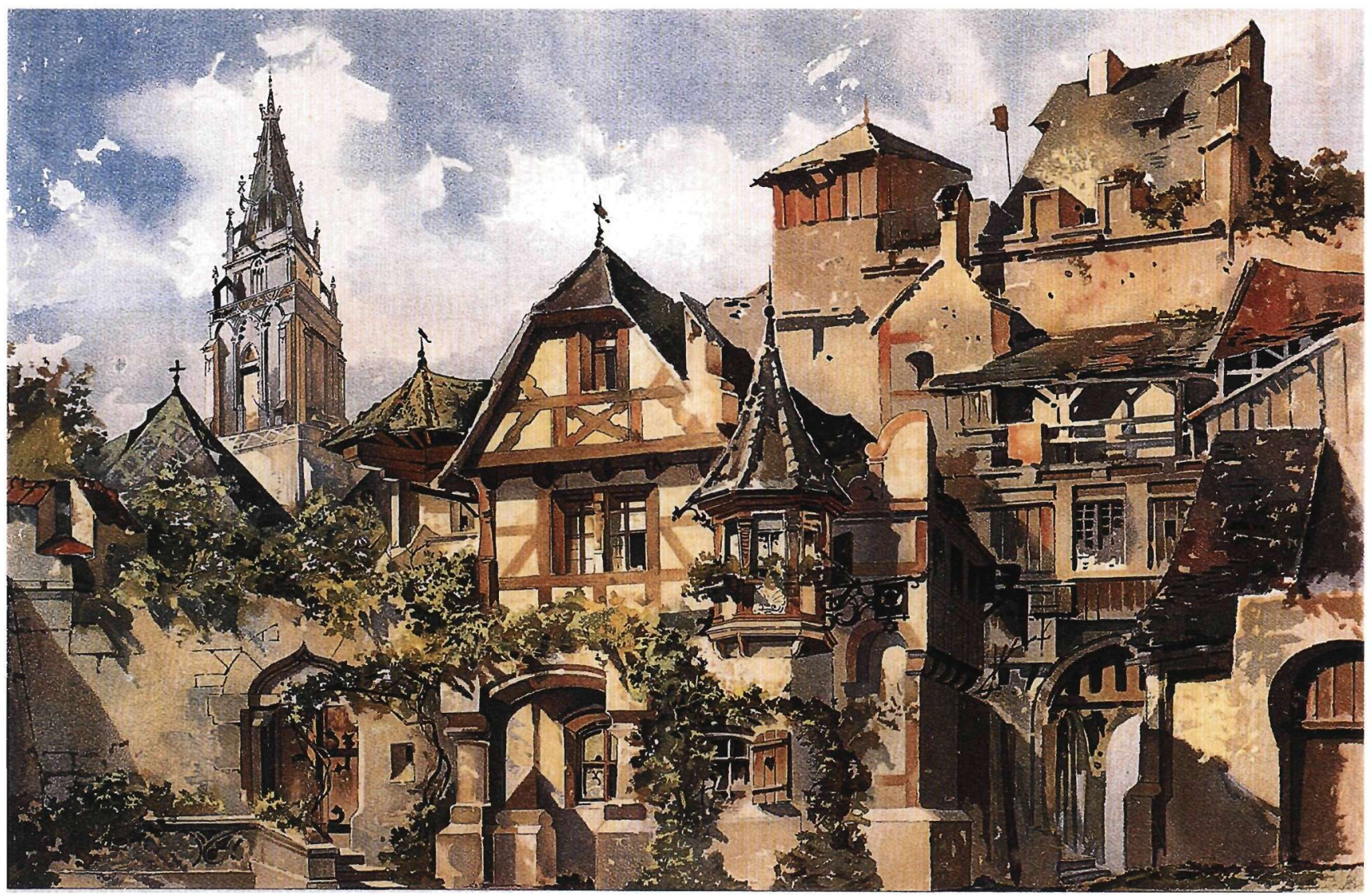

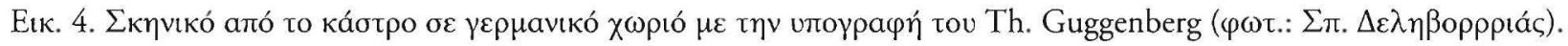

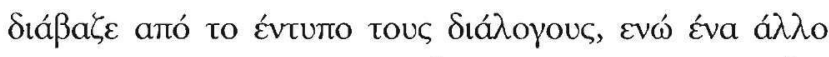

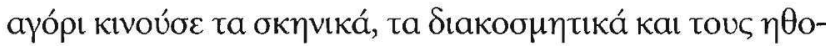

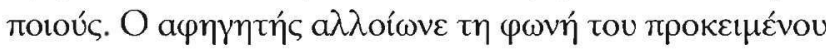

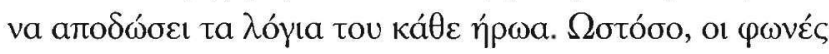

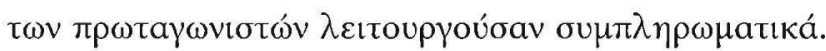

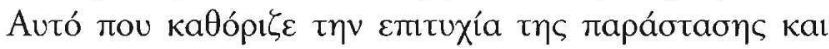

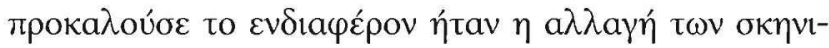

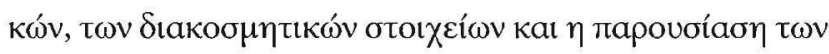
$\eta \theta$ oлоเ

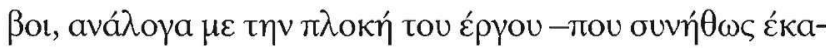

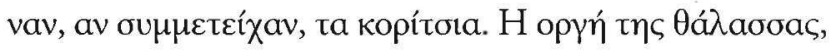

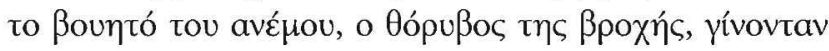

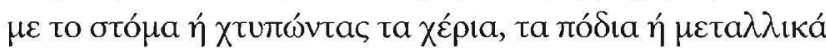

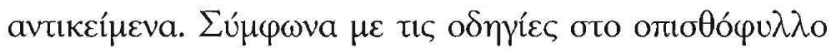

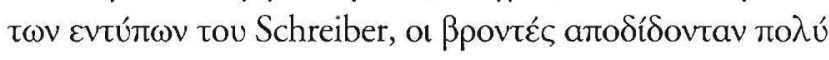

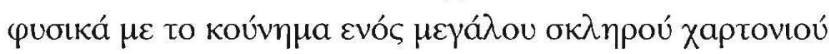

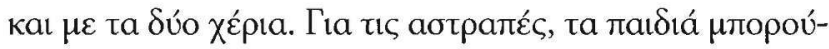

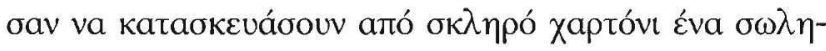

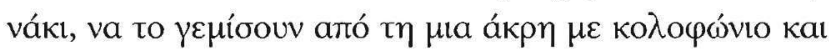

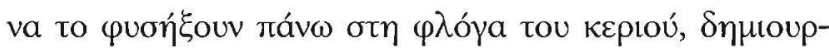

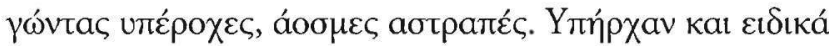

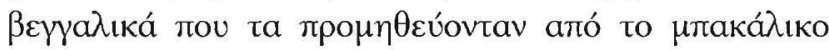

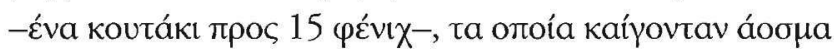

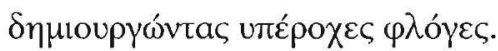

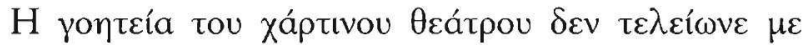

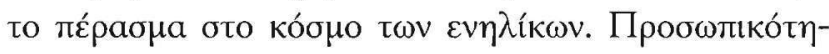

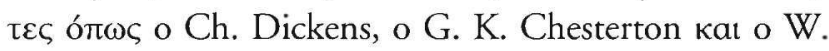

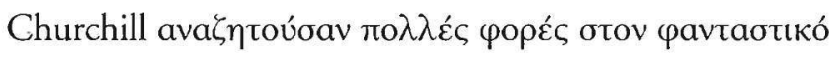

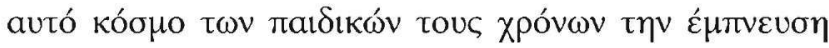
yıa to épjo tous. ${ }^{16}$

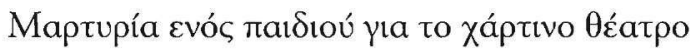

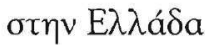

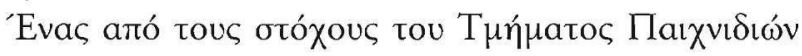
кaı Паı

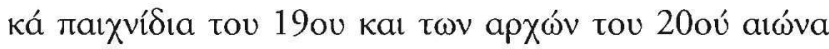

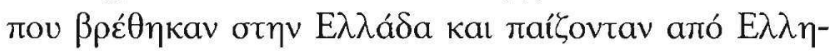

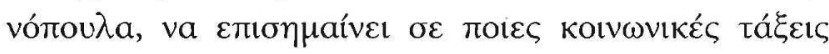




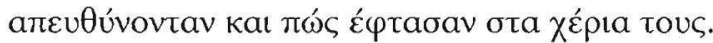

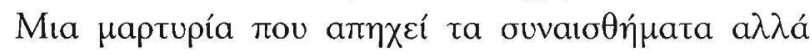

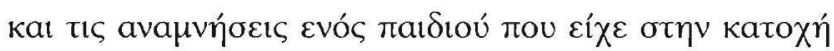

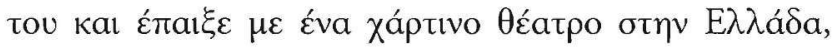

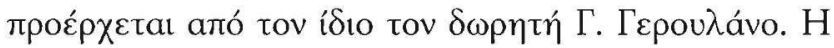

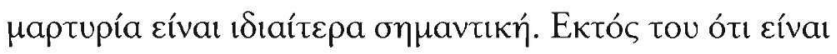
a

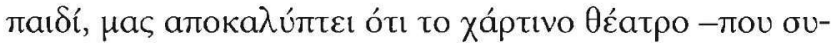

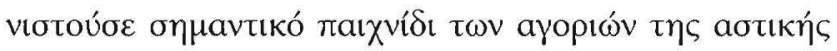

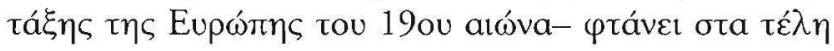

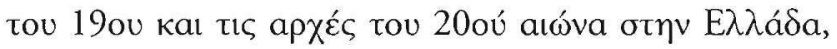

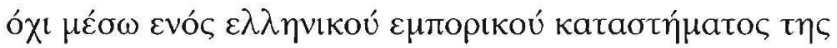

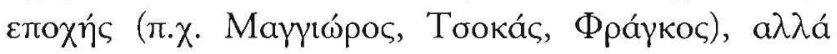

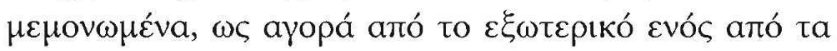

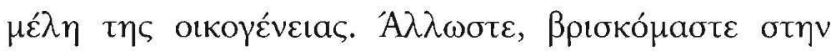

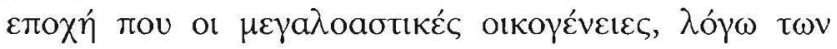

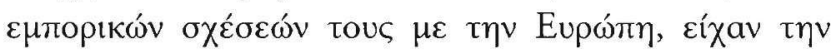

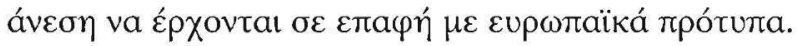

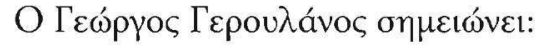

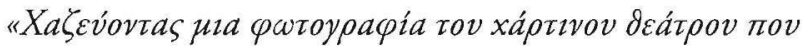

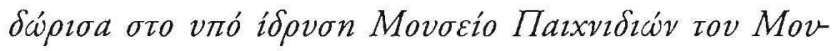

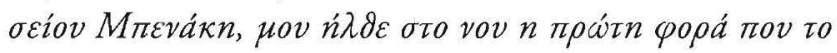
avtíkpioa.

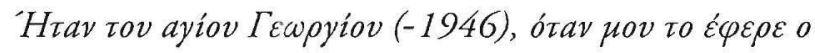

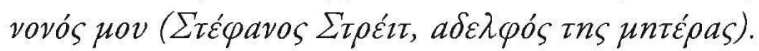

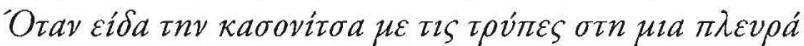

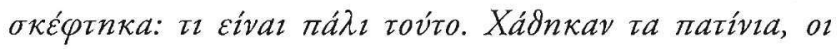

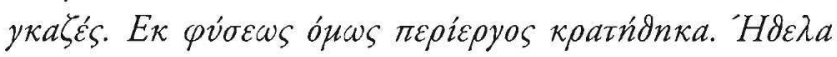

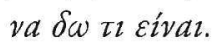

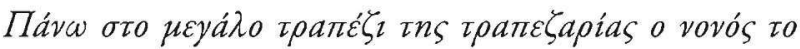

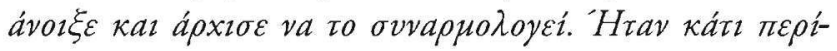

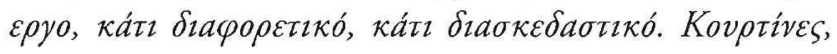

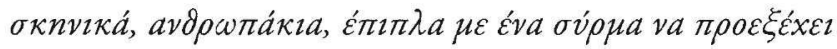

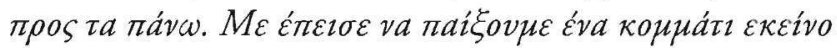

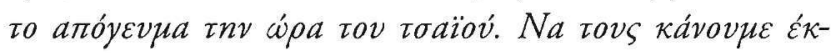

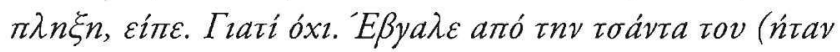

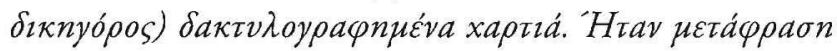

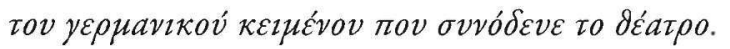

A

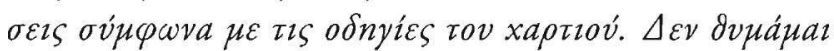

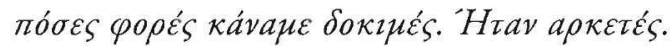

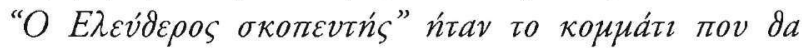

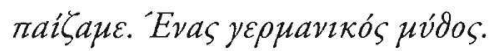

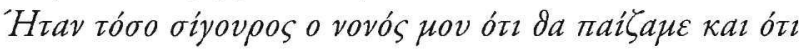

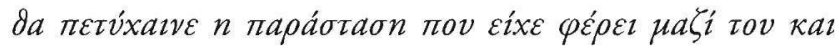

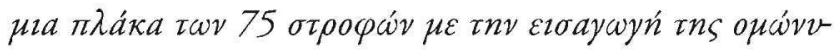

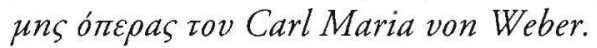

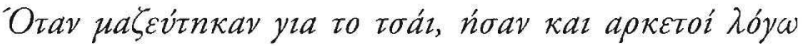

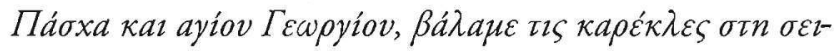

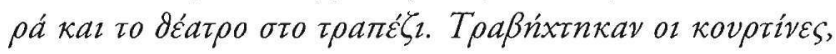

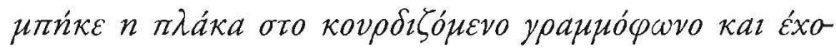

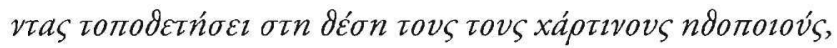

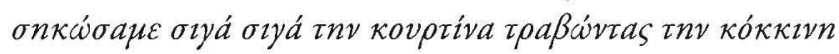

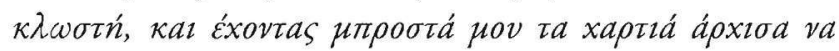

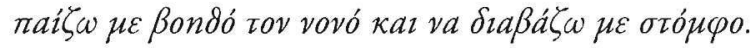

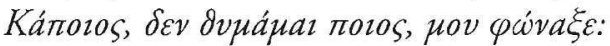

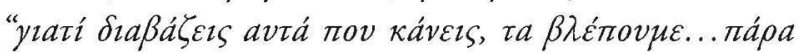
кárw".

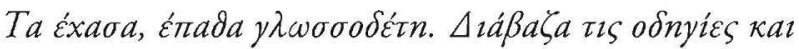

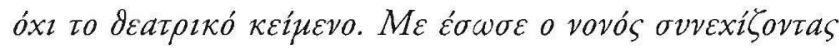

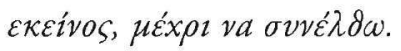

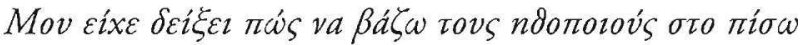

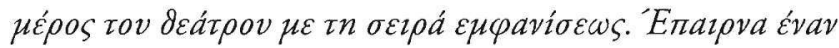

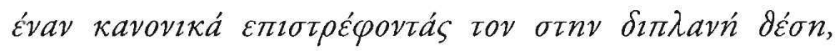

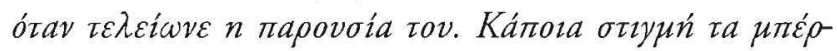

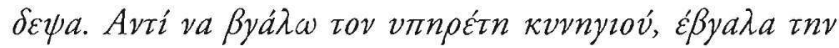

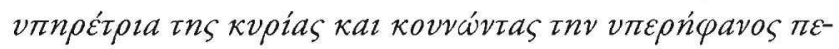

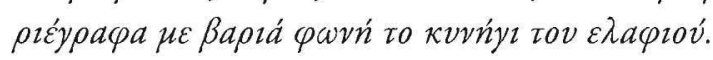

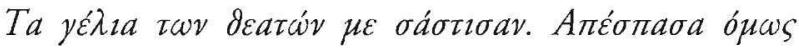

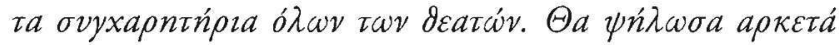

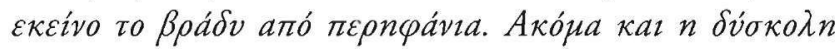

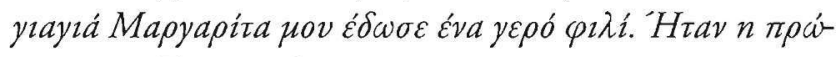

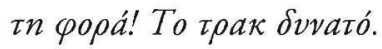

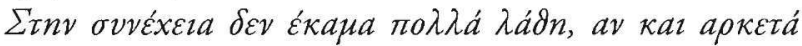

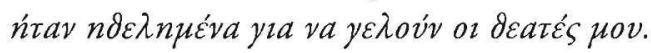

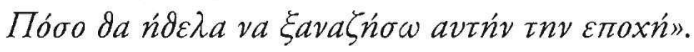

Mapía Apүuptáoๆ

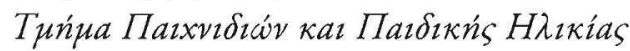

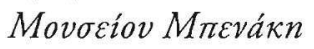




\section{$\sum H M E I \Omega \Sigma E I \Sigma$}

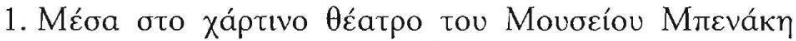

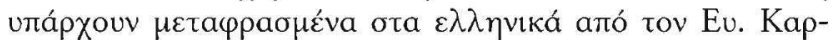

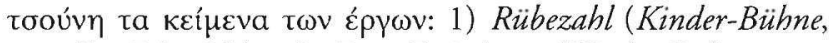
von R. Schmidt), 2) Der Freischütz (Kinder-Bühne, von Richard Schmidt), 3) Dornröschen (Kinder-Buhne, von R. Schmidt), 4) Aschenbrodel (Schreibers Kinder-Theater, von E. Sievert), 5) Die Puppenfee (Schreibers Kinder-Theater, von E. Sievert), 6) Rotkäppchen (Schreibers Kinder-Theater, von E. Sievert), 7) Schneewittchen und die sieben Zwerge (Schreibers Kinder-Theater, von E. Sievert).

2. Der Freischütz, óлєра тov C. M. von Weber.

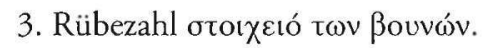

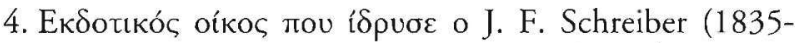

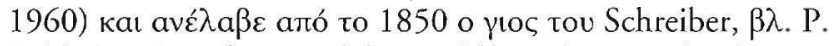
Baldwin, Toy Theaters of the World (London 1992) 86-98.

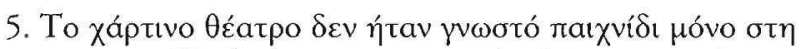

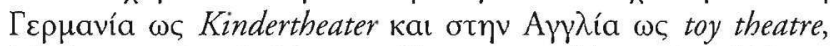

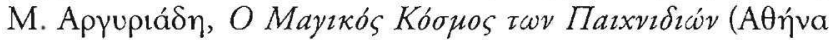

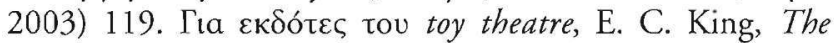
Encyclopedia of Toys (London 1978) 224-27. 'О $\pi \omega \varsigma$ हmơ-

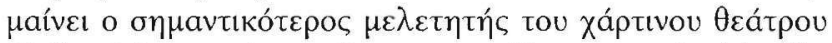

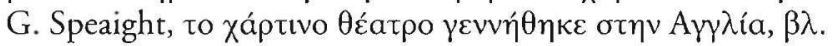
охعтाкá G. Speaight, The History of the English Toy Theatre

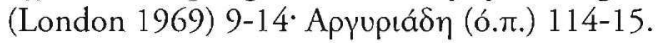

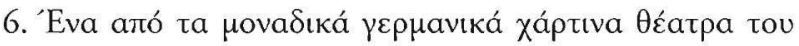

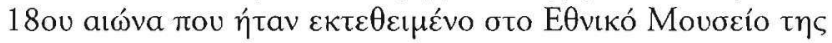

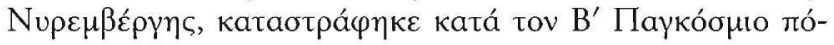

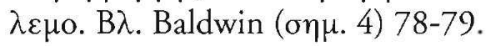

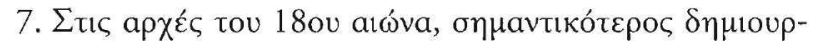

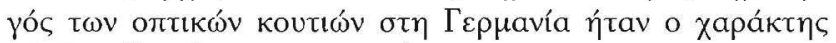

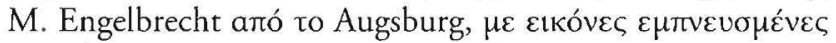

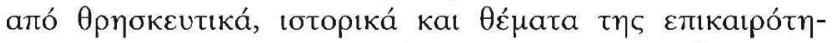

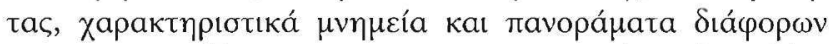
$\pi \varepsilon \rho$ เох

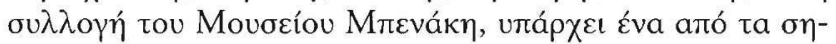

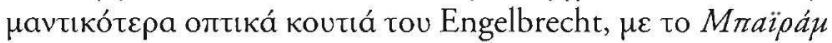

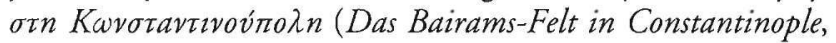

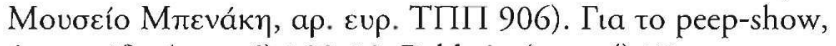

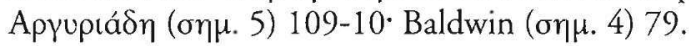

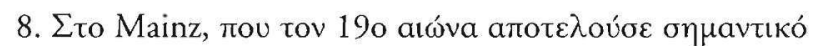

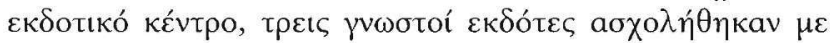

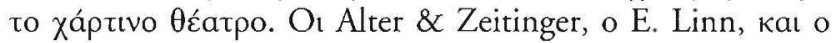

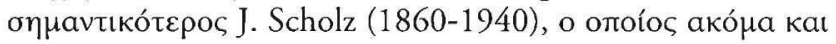

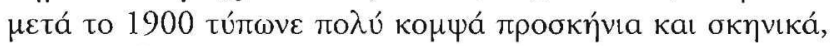

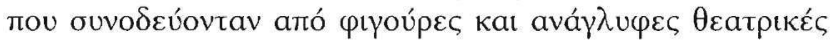

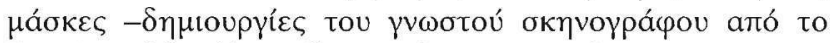

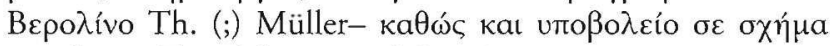

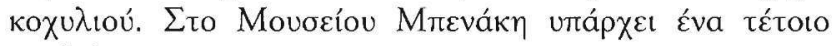

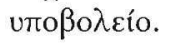

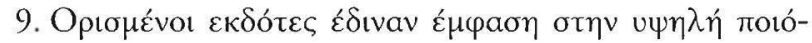

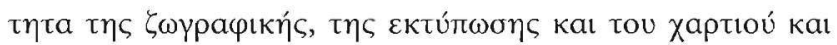

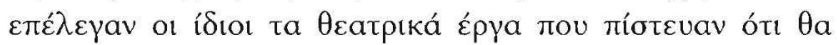

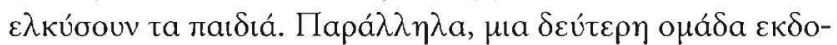

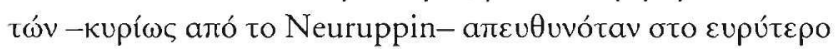

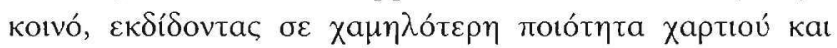

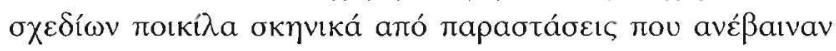

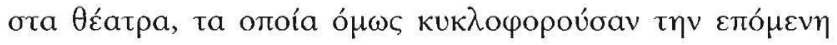

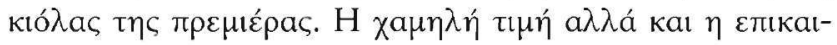

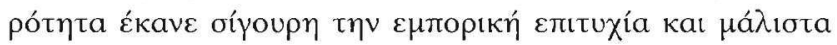

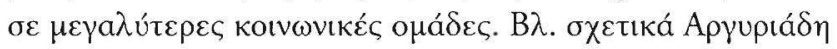
(оๆн. 5) 116-18.

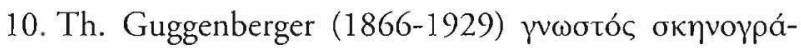

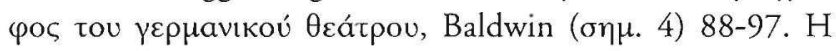

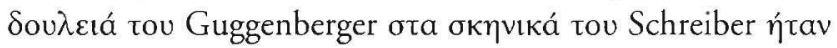

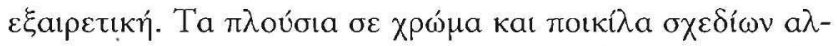

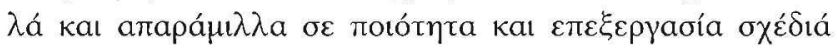

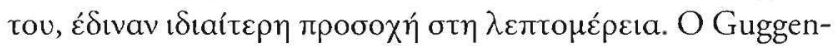

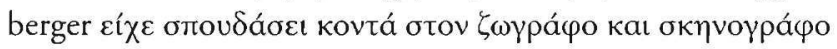

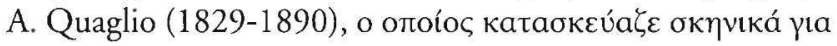

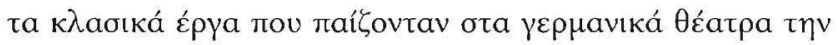

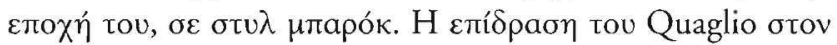

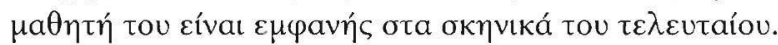

11. Baldwin $(\sigma \eta \mu .4) 79-98$.

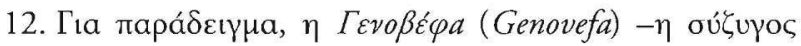

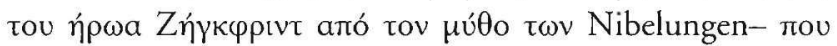

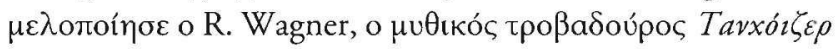

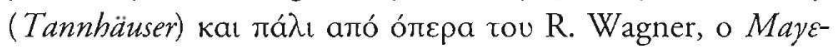

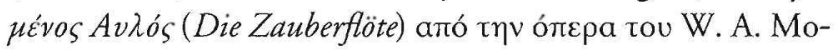

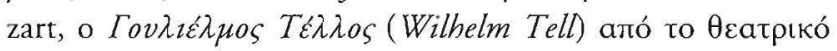
ع́pүo tou Fr. von Schiller.

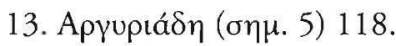

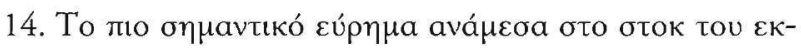

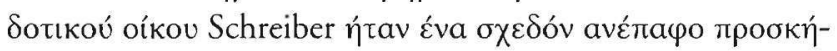

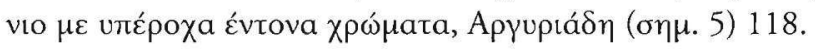

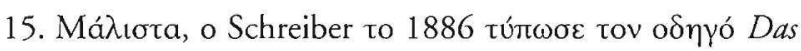

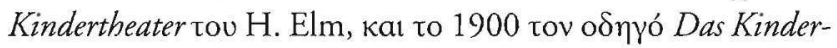

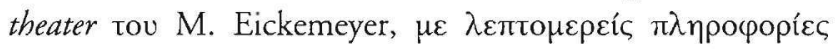

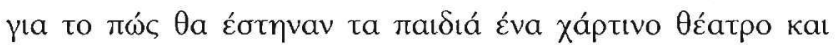

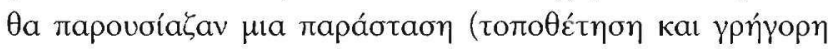

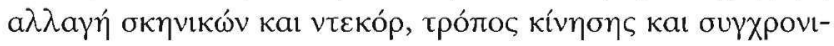

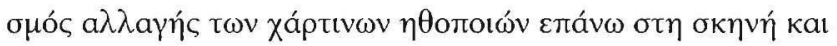

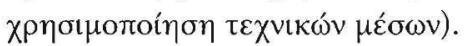

16. Speaight $(\sigma \eta \mu .5) 181-82$. 


\section{MARIA ARGYRIADI}

A German toy theatre (Papiertheater) from Schmidt \& Romer of Leipzig

This cut-out toy theatre (Papiertheater), manufactured by Schmidt \& Romer of Leipzig in 1890 , came into the possession of the Benaki Museum in 2000, as a donation from Georgos Yeroulanos, whose godfather had given it to him as a present after a visit to Germany in the 1940s.

It consists of a wooden box, which forms the base of the theatre and has holes for inserting the cardboard scenery. Inside the box are the cardboard proscenium with it inscription Thaleia after the Muse of Comedy, 10 large lithographed pieces of scenery with the side pieces facing each other and in perspective, various items of décor for interior scenes, the figures of the characters in board and seven leaflets with the scripts of plays.

The publishing house of R. Schreiber, which took over the business of Schmidt \& Romer, reproduced its work and its style, and even used its scenery, décor for interior scenes and cut-out characters. Schreiber's coloured lithographs for the scenery bring to mind scenes from fairy tales and children's literature and illustrations from the repertoire of German theatre dramatisations for children.
In the 19 th century most middle-class boys had a cutout theatre. They spent several days constructing and setting it up and then putting on a performance. Often the preparations took as long as a month. The first step was to buy the printed material which contained the script and the characters of the play. All the coloured lithographs for the scenery were obtained separately on large sheets from stationery shops.

The children cut out the scenery and the figures and stuck them on to pieces of board. The three-dimensional scenery was inserted in the holes in the wooden base. After several rehearsals, two or three children -mainly boys- presented the show. The red curtain was raised and the show began with some form of musical accompaniment. Behind the scenes the narrator read the dialogue aloud from the printed script, while another dealt with the scenery, the props and the actors. The dialogue provided for each character, which the narrator differentiated by a change of voice, represented an additional means of communication. 